\title{
Evolution and conservation of immunological activity
}

N.M. Vaz

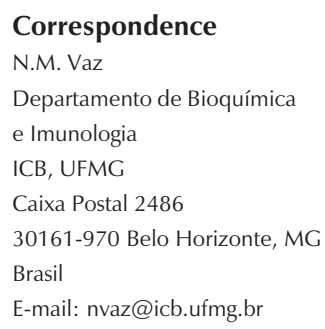

Received July 1, 2006 Accepted September 26, 2006 .....................
Departamento de Bioquímica e Imunologia, Instituto de Ciências Biológicas, Universidade Federal de Minas Gerais, Belo Horizonte, MG, Brasil
"Steps to an Ecology of Mind" (1) is an outstanding collection of essays and lectures by Gregory Bateson on a wide array of subjects, gathered by his students and associates. The book is divided into six sections and section IV, which is devoted to "Biology and Evolution", starts with a remarkable little text in which Bateson uses his wide culture to mock the anti-evolutionary ruling of the State Board of Education in California that, in 1970, demanded religion to be taught together with evolutionary principles. In this short paper, Bateson stated that:

"My father, the geneticist William Bateson, used to read to us passages of the Bible at breakfast - lest we grow up to be emptyheaded atheists; so I find it natural to wonder what broadening of the mind may come from the strange anti-evolutionary ruling of the State Board of Education in California." $\mathrm{He}$ also stated that: "Evolution has long been badly taught. In particular, students - and even professional biologists - acquire theories of evolution without any deep understanding of what problems these theories attempt to solve. They learn but little of the evolution of evolutionary theory."

He then suggests, ironically, that the State Board of Education may be right in forcing the students to study (Christian) religion because: "The extraordinary achievement of the writers of the first chapter of Genesis was their perception of the problem: Where does order come from? They observed that the land and the water were, in fact, separate and that the species were separate; they saw that such separation and sorting in the universe presented a fundamental problem. In modern terms we may say that this is the problem implicit in the Second Law of Thermodynamics: If random events lead things to getting mixed up, by what non-random events did things come to be sorted? And what is a "random" event?"

Bateson insists that the problem of order, which is not trivial, has been central to Biology and to many other sciences for the last 5000 years, and asks: "With what word should we designate the principle of order which seems to be immanent in the Universe?" In his irony, Bateson says that the California ruling might get students to consider differ- 
ent answers to this ancient problem: "Our students might have their minds broadened somewhat if they would look at other theories of evolution and consider how a man's spirit must take a different shape if he believes that all sorting in the universe is due to an external agent, or if, like the Iatmul and modern scientists, he sees that the potentiality for order and pattern is immanent throughout this world."

The Iatmul are a tribe of Stone Age head hunters from a swampy region in New Guinea who believe that land was separated from water when a vast crocodile, Kavwokmali, that paddled his back legs and maintained the mud in suspension, was killed by the culture hero, Kevembuangga (1).

The contrast between transcendent and immanent solutions to the origin of order should be perceived as the main issue in Evolution, but this is certainly not the general trend, and Wilkins (2) may be correct when stating that: "The subject of evolution occupies a special, and paradoxical, place within biology as a whole. While the great majority of biologists would probably agree with Theodosius Dobzhansky's dictum that "nothing in biology makes sense except in the light of evolution", most can conduct their work quite happily without particular reference to evolutionary ideas. "Evolution" would appear to be the indispensable unifying idea and, at the same time, a highly superfluous one."

To which Conway Morris (3) might have added: "When discussing organic evolution the only point of agreement seems to be: "It happened." Thereafter, there is little consensus, which at first sight must seem rather odd."

Maturana and Mpodozis (4) are two other biologists who claim that the main issue in Evolution is not generally acknowledged, but they add an important second twist. They claim to be proposing a basic conceptual change in dealing with the same fundamental questions that led Darwin to the theory of evolution by means of natural selection, because:

“...change occurs continuously as a spontaneous feature of the molecular existence of living systems (and of all molecular systems), and that as an intrinsic condition of the existence of living systems it must not be explained. What must be explained is the course that change follows in the ontogeny and phylogeny of living systems."

They say that the most important concept in the evolutionary process is not to know how living beings are diversified or conserve their characteristics of their adaptation to their circumstances, because: "this is already understood, at least in general lines". What must be understood is how this variation and this conservation followed the particular trend they followed. How did living beings vary the way they did, and how did they conserve invariant certain aspects of living and not others (4)?

Conservation and variation of form was the major concern of Gregory Bateson's father, William Bateson, who published a remarkable collection of observations on the subject (Bateson, 1894), recently reprinted by the John Hopkins University Press (5). In the introduction to this book, Webster (6) quotes: "In a summary of his conclusions, (William) Bateson rejects "the crude belief that living systems are plastic conglomerates of miscellaneous attributes and that order or form or Symmetry have been impressed upon this medley by Selection alone" (p. 80, of Variation). Rather, he suggests, we have to recognize that "the system of an organized being is such that the result of its disturbance may be specific" (p. 74). "This remarkable conclusion implies that the response, in terms of the form produced, of a biological system to perturbation - the action of a causal agent - is determined by its intrinsic repertoire of possibilities, that is, the inherent nature of the particular causal mechanism implicated."

Thus, here is a clear statement by Gre- 
gory Bateson's father and predecessor declaring his faith in immanent mechanisms in the generation of biological form. Webster (6) proceeds to mention the phenomenon of phenocopying in support of William Bateson's idea: "Perhaps the most striking support for Bateson's conclusion is provided by the phenomenon of phenocopying investigated by Goldschmidt (7) who claims that practically any kind of environmental perturbation of developing wild-type Drosophila, applied at the appropriate time, will result in a specific morphology which is normally associated with the presence of a mutant allele." Goldschmidt also discusses these phenomena in terms of the range of forms, which is inherently possible for the organism and which "determines the possibility of appearance of both mutational and environmental effects."

\section{Change and conservation in immunology}

Recently (8-10) and also not so recently (11), I have insisted that, to be treated genuinely as a system, the immune system must be endowed with a defined organization, i.e., an invariant set of relations among some of its components (12). There is abundant evidence for the generation of (clonal) diversity (GOD) during lymphopoiesis $(13,14)$. In contrast, the still scarce evidence for conserved patterns of operation in immunological activities has remained unacknowledged.

Nevertheless, a significant number of observations on the production of natural plasma immunoglobulins, i.e., those appearing in healthy organisms without artificial immunization, has shown that, early in ontogeny, their reactivity is spontaneously organized in patterns (15) that are influenced by genes important for immunological activity (16) but, surprisingly, not by contact with environmental antigens, such as those derived from food and flora (17), and are robustly conserved throughout healthy liv- ing (18). In other words, these observations point to mechanisms of organization in the immune system which are immanent to its operation. Immunoglobulins formed by newborn mice are known to favor an internal interconnectivity (19) and interference with their formation is known to cause severe abnormalities in the adult animal (20). Furthermore, the several aspects of processes leading to lymphocyte activation have been shown to be conducive to the generation of lymphocyte interconnections (21).

There is a large disproportion between the immense amount of information available on the nature and operation of genetic/ cellular/molecular components of the immune system and the possibility to extract from it practical, clinical, diagnostic, and preventive applications. The impediments to this development are conceptual rather than technological and lie in the way of seeing immunological activity as a form of response to foreign materials.

Since the variety of foreign materials which may eventually invade the organism is virtually unlimited, a corresponding unlimited variety of lymphocytes would be necessary to cope with the need to respond specifically to each one of them. The solution to this riddle was the adoption of a NeoDarwinian solution, i.e., coupling a random source of variants (lymphocyte clones) with a selective mechanism (competition among existing clones for binding of foreign materials) - which is the essence of the dominant immunological theories, all based on stimulus/response/regulation models $(13,14)$.

The observation of robustly conserved patterns of reactivity, as well as several other lines of evidence, rule out the possibility that immunological activity is generated at random and calls for historic/systemic descriptions. Paraphrasing Maturana and Mpodozis (4), the main issue to be explained in the ontogeny of immunological activity is not how lymphocyte diversity is generated, because this is already known, at least in gen- 
eral lines and, as they say, change is an intrinsic condition of existence in living systems and this does not need to be explained. "What must be explained is the course that change follows in the ontogeny and phylogeny of living systems" (4). Therefore, the deep issues in Immunology are: "How did the combinatorial immune system arise in phylogeny the way it did?" and "How does the immune system organize itself in ontogeny the way it does, and from then on, conserves its pattern of reactivity?"

\section{References}

1. Bateson G. An empty-headedness among biologists and State Boards of Education. In: Bateson G (Editor), Steps to an ecology of mind. New York: Balantine Books; 1973. p 343-345.

2. Wilkins AS. Evolutionary processes: a special issue. Bioessays 2000; 22: 1051-1052.

3. Morris SC. Evolution: bringing molecules into the fold. Cell 2000; 100: 1-11.

4. Maturana $\mathrm{H}$, Mpodozis J. The origin of species by means of natural drift. Rev Chilena Hist Nat 2000; 73: 261-310.

5. Bateson W. Materials for the study of variation: treated with special regard to discontinuity in the origin of species (original edition 1894). Mabee PM, Fitzhugh K (Editors), Foundations of Natural History. Baltimore and London: John Hopkins University Press; 1992.

6. Webster G. William Bateson and the science of form. In: Mabee PM, Fitzhugh K (Editors), Bateson W. Materials for the study of variation treated with special regard to discontinuity in the origin of species (original edition 1894). Foundations of Natural History. Baltimore and London: John Hopkins University Press; 1992. p 49-50.

7. Goldschmidt R. The material basis of evolution. New Haven: Yale University Press; 1940.

8. Vaz NM. The cellular and molecular correlates of immunological phenomena. Contrasting two explanatory pathways. Braz J Med Biol Res 1996; 26: 545-553.

9. Vaz NM, de Faria AM, Verdolin BA, Silva Neto AF, Menezes JS, Carvalho $\mathrm{CR}$. The conservative physiology of the immune system. Braz J Med Biol Res 2003; 36: 13-22.

10. Vaz NM. Immunology: elementary concepts and misconceptions. Proceedings of the Third Brazilian Symposium of Mathematical and Computational Biology. Rio de Janeiro: 2004. p 412-431.
11. Vaz NM, Varela FJ. Self and non-sense: an organism-centered approach to immunology. Med Hypotheses 1978; 4: 231-267.

12. Maturana $\mathrm{H}$. Autopoiesis, structural coupling and cognition: a history of these and other notions in the biology of cognition. Cybernetics and Human Knowing 2002; 9: 5-34.

13. Abbas AK, Lichtman A. Cellular and molecular immunology. 5th updated edn. New York: WB Saunders Company; 2005.

14. Janeway CA. Immunobiology: the immune system in health and disease. New York: Current Biology; 2001.

15. Nobrega A, Stransky B, Nicolas N, Coutinho A. Regeneration of natural antibody repertoire after massive ablation of lymphoid system: robust selection mechanisms preserve antigen binding specificities. J Immunol 2002; 169: 2971-2978.

16. Vasconcellos R, Nobrega A, Haury M, Viale AC, Coutinho A. Genetic control of natural antibody repertoires: I. $\mathrm{IgH}, \mathrm{MHC}$ and TCR beta loci. Eur J Immunol 1998; 28: 1104-1115.

17. Haury M, Sundblad A, Grandien A, Barreau C, Coutinho A, Nobrega $A$. The repertoire of serum IgM in normal mice is largely independent of external antigenic contact. Eur J Immunol 1997; 27: 1557-1563.

18. Lacroix-Desmazes S, Mouthon L, Kaveri SV, Kazatchkine MD, Weksler ME. Stability of natural self-reactive antibody repertoires during aging. J Clin Immunol 1999; 19: 26-34.

19. Stewart J, Varela FJ. Exploring the meaning of connectivity in the immune network. Immunol Rev 1989; 110: 37-61.

20. Vakil M, Sauter H, Paige C, Kearney JF. In vivo suppression of perinatal multispecific $B$ cells results in a distortion of the adult $B$ cell repertoire. Eur J Immunol 1986; 16: 1159-1165.

21. Vaz NM, Faria AMC. The construction of immunological identity. Cienc Cult 1990; 42: 430-444. 